\title{
Evaluación de productos para el control de polvo ambiental en caminos mineros
}

\section{Products evaluation for controlling enviroenmental dust in mining roads}

\author{
Diego Andrés Brauer ${ }^{1 *}$, Andrea Alejandra Giubergia ${ }^{1}$, Verónica Gil-Costa ${ }^{1,2}$ \\ ${ }^{1}$ Universidad Nacional de San Luis, Argentina \\ ${ }^{2}$ CCT-SL(CONICET-CCT), San Luis, Argentina \\ *Autor para la correspondencia: aagiuber@unsl.edu.ar
}

\begin{abstract}
Resumen
El estudio tuvo la finalidad de evaluar diferentes técnicas para lograr disminuir el polvo suspendido en caminos mineros. Luego de una búsqueda de información para lograr este propósito se experimentó con cinco productos: melaza, hidróxido de magnesio, TGC, cloruro de sodio y bischofita, algunos de ellos aplicados con este fin en caminos. Con asistencia del software Comet y el equipo captador de polvo E-Sampler se recolectaron datos de mediciones de polvo y se compararon con datos previos tomados por especialistas en medio ambiente. El producto bischofita revela los mejores resultados en las zonas tratadas previamente con suelosal, sin embargo, no es posible aplicarlo durante las épocas de nevada por la inseguridad que genera al tornar muy resbaloso el camino.
\end{abstract}

Palabras clave: bischofita; melaza; control de polvo; caminos mineros; software Comet.

\section{Abstract}

The purpose of the study was to evaluate different techniques for reducing suspended dust in mining roads. After searching for some information to fulfill this purpose this purpose we experimented with five products such as: molasses, magnesium hydroxide, TGC, sodium chloride and bischofit, some of them applied for this purpose on roads. Dust measurement data were collected and compared with previous data taken by environmental 
specialists and the E-Sampler dust collection device by using the assistance of the Comet software. The bischofit product revealed the best results in the areas previously treated with soil-salt; however it is not possible to apply it during the snowy season because of the insecurity it generates when the road becomes very slippery.

Keywords: bischofit; molasses; dust control; mining roads; Comet software.

\section{INTRODUCCIÓN}

Las operaciones mineras movilizan grandes cantidades de material que requieren maquinaria pesada y equipos industriales para procesar el mineral. Las pilas o depósitos de desechos contienen partículas pequeñas que pueden ser fácilmente dispersadas por el viento. Las mayores fuentes de contaminación del aire en operaciones mineras son:

- Material particulado transportado por el viento como resultado de excavaciones, voladuras, transporte de materiales, erosión eólica (más frecuente en minas a cielo abierto), polvo proveniente de los depósitos de relaves, depósitos, pilas de desechos, caminos. Las emisiones de los gases de escape de fuentes móviles (vehículos, camiones, maquinaria pesada) también contribuyen a aumentar el nivel de material particulado.

- Emisiones gaseosas provenientes de la quema de combustibles en fuentes estacionarias como móviles, voladuras y procesamiento de minerales.

Cuando una fuente emite contaminantes a la atmósfera estos son transportados en el aire, se diluyen y son sujetos a cambios (físicos y químicos) en la atmósfera y finalmente alcanzan al receptor (personas, vehículos de transportes). Estos contaminantes pueden causar serios efectos en la salud de las personas y en el ambiente (Instituto Geológico y Minero de España 2004; Yang y Lin 2009; Ospina, González y Fernández 2011).

La minería a gran escala puede contribuir potencialmente a la contaminación del aire, especialmente en el transcurso de la etapa de operación. Las actividades durante la extracción de mineral, procesamiento, manipulación y transporte dependen del equipo, del tipo de generadores de energía, procesos y materiales que pueden generar contaminantes atmosféricos peligrosos, tales como material particulado, metales pesados, 
monóxido de carbono, dióxido de azufre y óxidos de nitrógeno. Sin un adecuado sistema de control de polvo, este material particulado afectará negativamente el desempeño de los equipos de producción, lo que compromete la productividad de la operación y perjudica la salud de las personas (Tapia 2017; Rada-Jaman 2017).

Un camino que no levante polvo utiliza menos agua para su mantención y mejora la carpeta de rodado (Beaulieu et al. 2011; Urteaga 2016; UlateCastillo y Vargas-Sobrado 2018; Guivar-Pereda y Zelada-Alaya 2018). Por lo tanto, los vehículos consumen menos combustible, se extiende la vida útil de los neumáticos y entrega un entorno más seguro. Además, mejora la calidad de vida para las personas y las comunidades aledañas, dejando de afectar la salud de trabajadores y vecinos (Addo, Sanders y Chenard 2004).

Tradicionalmente la mayoría de las mineras mitigan por aglomeración de partículas de polvo por peso y humedad, regando los caminos (GastañagaColl y Medina 1963; Hernández 2018). Esta práctica es ineficiente e ineficaz, ya que las tasas de evaporación en zonas áridas y calurosas son de 30 min aproximadamente $y$, en climas fríos de montaña, se congela y genera resbalamiento.

Igualmente, hay productos que son altamente corrosivos, se tornan jabonosos, lixivian, filtran y promueven la migración de los agentes químicos al medio ambiente, cambian el $\mathrm{pH}$ del medio acuoso y napas subterráneas, por mencionar algunos problemas. Esto no sucede con las emulsiones, cuyo componente bituminoso es superior al $60 \%$.

Gutiérrez-Montes (2010) realiza un estudio sobre las propiedades de los suelos tratados con sal magnesio (bischofita), donde cumple un papel muy importante en la estabilización de los suelos y menciona casos de uso con resultados satisfactorios.

Existen diferentes tipos de supresores de polvo que contribuyen a hacer efectivo el control de polvo, como lo describen los trabajos de VergaraRavanal (2011), López-Chegne (2012) y Ovalle-Cárdenas (2014). En estos estudios se destacan los productos a base de:

- agua, la cual es una solución de corto plazo y opera a partir de la aglomeración de partículas en la superficie;

- sales y cloruros, que suprimen polvo a partir de la atracción de la humedad del ambiente y son más efectivos en lugares que tienen alta humedad y bajas temperaturas; 
- polímeros sintéticos que pueden fortalecer la superficie del camino, aumentando la tensión de rotura en diez veces;

- productos orgánicos no bituminosos, actúan como cementante, ligando las partículas del suelo entre sí. Tienden a mantener la plasticidad, permitiendo la compactación del suelo cuando son aplicados en caminos con alto contenido de arcilla;

- productos bituminosos que incluyen los asfaltos cortados y emulsiones asfálticas, entre otros; y

- productos electroquímicos que son usualmente derivados de petróleo sulfatado y productos altamente iónicos. Se incluyen en este grupo los aceites sulfatados, enzimas y cloruro de amonio.

Este trabajo presenta un estudio de diferentes métodos, según el producto utilizado para mitigar el nivel de polvo en los pisos de los caminos mineros por los que transitan vehículos de distinto porte para una mina a cielo abierto de oro y plata de Argentina, realizado entre los años 2012 y 2015. La mina se encuentra en la zona cordillerana de Argentina (Sistema de Información Geológica Ambiental Minera SIGAM 2018), donde es frecuente encontrar hielo y nieve en los caminos (Pradena, Mery y Novoa 2010).

El software Comet se utiliza junto con el captador de polvo E-Sampler para obtener la descripción y el análisis de resultados. Los caminos en los cuales se estudian las aplicaciones de aditivos se dividen en caminos destinados a transporte de personal y caminos destinados al transporte de mineral. La circulación de vehículos en caminos de transporte de personal es principalmente de camionetas y buses, mientras que en caminos destinados al transporte de mineral la circulación es de camiones CAT 793, los cuales tienen un peso aproximado de $200 \mathrm{~T}$ y la carga máxima a transportar es de 240 T. También circulan equipos auxiliares como motoniveladoras, topadoras sobre orugas, topadoras sobre neumáticos y otros.

\section{MATERIALES Y MÉTODOS}

\subsection{Melaza}

La melaza es un producto orgánico derivado de la caña de azúcar que se utiliza frecuentemente como fertilizante en la industria agropecuaria. Esta es una sustancia estable y no reactiva, no presenta condiciones indeseables ni materiales o productos de descomposición ni polimerización.

Existe un derivado industrial de este producto, llamado SMARTER V55, desarrollado para el tratamiento de caminos, ya que por sus características 
adhesivas se comporta como excelente estabilizador de suelo y supresor de polvo.

Con respecto a la información toxicológica, la solución no presenta efectos al entrar en contacto con la piel y los ojos. En caso de que esto ocurra o se produzca por accidente ingestión o inhalación, se sugiere enjuagar con abundante agua. No es combustible ni inflamable y no presenta riesgo de explosión. Debido a lo anterior cabe destacar que no exige condiciones especiales para manipulación, transporte ni almacenamiento.

Para las pruebas de melaza, primero se verificó que la solubilidad del producto fuese buena para excluir problemas de dilución con el agua en el interior del tanque del camión destinado a los riegos. La solución se desarrolló con 6000 I de agua y 1000 I de melaza (14\% en volumen) y se destinó al riego de bermas.

\subsection{Hidróxido de magnesio, $\mathrm{Mg}(\mathrm{OH})_{2}$}

El hidróxido de magnesio es un polvo blanco o granulado muy poco soluble en agua que se aplica comúnmente en la industria del caucho, aparatos eléctricos, plásticos de ingeniería, como materia prima de otras sales de magnesio, como fertilizante, para neutralizar aguas residuales ácidas, como aditivo en el petróleo, como laxante, etc.

En una botella, se disolvieron $400 \mathrm{~cm}^{3}$ de hidróxido de magnesio en dos litros de agua (20\%), lo que generó una densidad de $1,1 \mathrm{~g} / \mathrm{cm}^{3}$. Luego se agitó la botella durante más de 10 min para intentar realizar la disolución. Pasados 10 min de concluida la agitación la solución se separó en tres fases. En el fondo de la botella se encuentra una sedimentación, en el centro una región blanca grisácea densa y en la parte superior aproximadamente $3 \mathrm{~cm}$ de agua clara. Además, la densidad descendió a $1,09 \mathrm{~g} / \mathrm{cm}^{3}$. Luego de $20 \mathrm{~min}$ (Figura 1 ) de realizada la agitación la densidad disminuyó a $1,08 \mathrm{~g} / \mathrm{cm}^{3}$. 


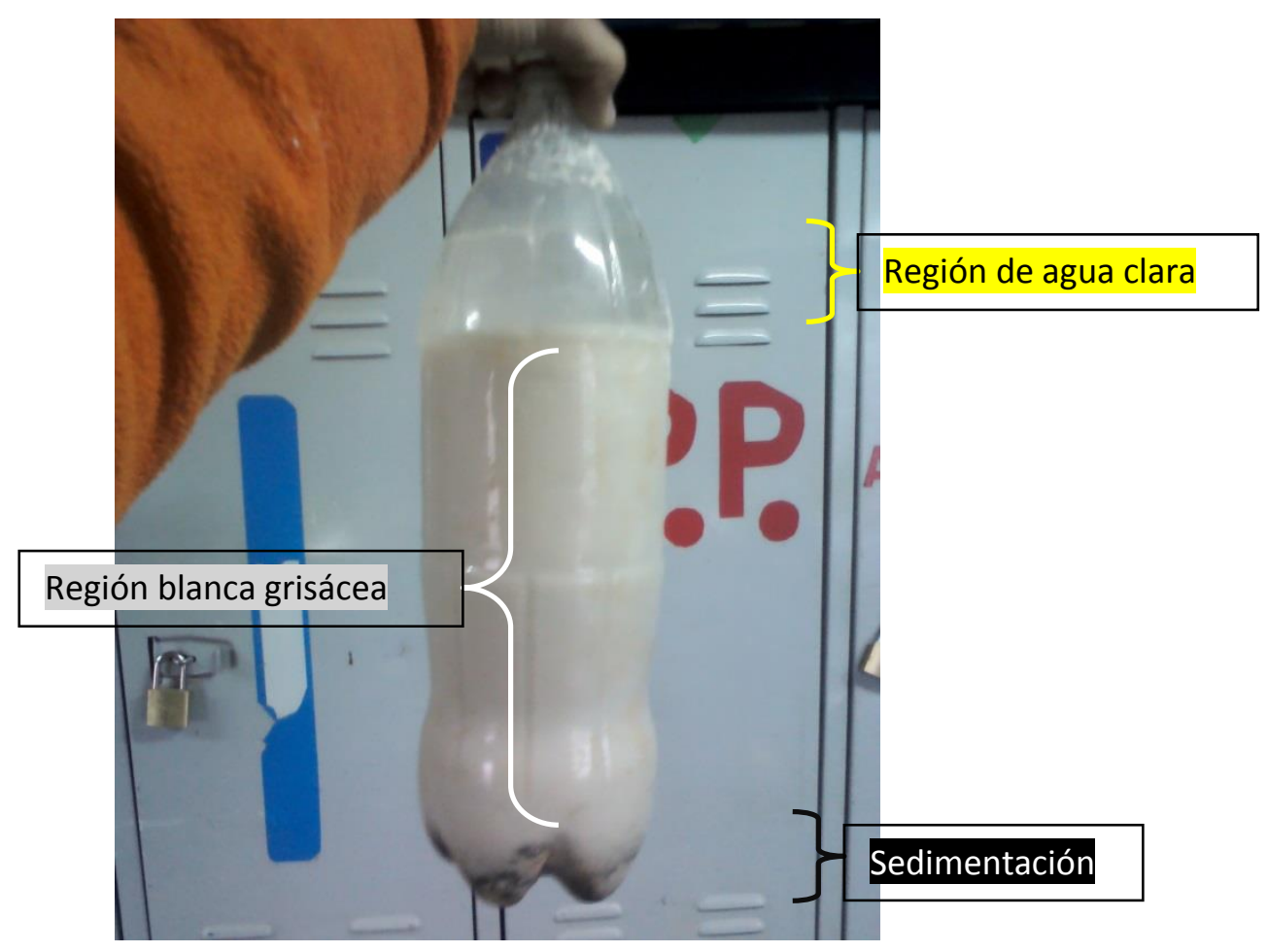

Figura 1. Solución luego de 20 min de la agitación.

En una segunda prueba se disolvieron $200 \mathrm{~cm}^{3}$ de hidróxido de magnesio en 2 I de agua (10\%), lo que generó una densidad de 1,03 g/cm ${ }^{3}$. El resultado fue que la sedimentación se produjo con mayor rapidez en la prueba con menor cantidad de hidróxido de sodio, bajando la densidad luego de los $10 \mathrm{~min}$ de la agitación a $1,02 \mathrm{~g} / \mathrm{cm}^{3}$. Además, pasados $10 \mathrm{~min}$ de la agitación la zona de agua limpia era de $6 \mathrm{~cm}$, mientras que habiendo transcurrido 20 min esta zona aumentó a $14 \mathrm{~cm}$, manteniéndose constante la densidad.

Al llevar la prueba a un volumen aún mayor, se realizó la disolución de $4000 \mathrm{~cm}^{3}$ de hidróxido en 15 I de agua (solución al $27 \%$ ), obteniéndose una densidad de $1,12 \mathrm{~g} / \mathrm{cm}^{3}$. Pasados $10 \mathrm{~min}, 20 \mathrm{~min}$ y $30 \mathrm{~min}$, la solución se mantuvo homogénea y conservó la densidad. Pero luego de $40 \mathrm{~min}$, se observó que se comenzó a producir sedimentación en el fondo del recipiente y se formó una pequeña capa de agua clara en la superficie de la solución.

Se hicieron otras pruebas de disolución de hidróxido de magnesio, pero con agua a alta temperatura, para constatar si de esta forma se lograba homogenizar la solución. A los 20 min de realizada la mezcla se notó que el recipiente con $200 \mathrm{~cm}^{3}$ de hidróxido contenía $21 \mathrm{~cm}$ de agua limpia y luego la capa de deposición del hidróxido de sodio; mientras que el recipiente con $400 \mathrm{~cm}^{3}$ de hidróxido, para el mismo tiempo de $20 \mathrm{~min}$, contenía $11 \mathrm{~cm}$ de agua limpia y luego la deposición del hidróxido. 


\subsection{TGC}

El producto TGC (Total Ground Control) es una emulsión de polímero desarrollado por la empresa RST como un agente generador de costras a largo plazo sobre cualquier tipo de material. La dilución debe realizarse en agua al 2-3 \% en volumen y su aplicación se debe hacer a una razón de $2 \mathrm{l} / \mathrm{m}^{2}$.

Con respecto a la información toxicológica, de entrar en contacto con la piel, boca $u$ ojos se recomienda el enjuagado con abundante agua y si el producto fuese inhalado mover a la persona expuesta al aire fresco. No presenta riesgo de explosión y en caso de incendio se recomienda utilizar agua, dióxido de carbono, espuma o medios secos de extinción. Debido a lo anterior cabe destacar que no exige condiciones especiales para manipulación, transporte ni almacenamiento.

Se probó la efectividad del TGC, utilizando $2 \mathrm{l} / \mathrm{m}^{2}$ y a una disolución de $3 \%$ (30 $\mathrm{ml}$ de TGC/litro de solución), la cual se aplicó en tres zonas de material fino, ubicadas en una escombrera o botadero de la mina.

\subsection{Cloruro de sodio, $\mathrm{NaCl}$}

Para aumentar la eficiencia de riego, se colocó previamente una carpeta de suelo-sal compuesta por $80 \%$ de coluvio clasificando como tamaño máximo de partículas de $3 / 4^{\prime \prime}$ y $20 \%$ de cloruro de sodio (se recomienda la humectación de la zona, previo al extendido de material). Se utilizaron camiones volcadores para su traslado y motoniveladoras para su extendido.

Luego de su extendido se recomienda humectar la zona por medio de camiones regadores, lo cual permite aumentar la penetración de la solución salina.

\subsection{Bischofita, $\mathrm{MgCl}_{2} \cdot 6 \mathrm{H}_{2} \mathrm{O}$}

La bischofita es una sal de magnesio soluble y es el método por excelencia para la estabilización de caminos. Se aplica en casi todas las faenas de Chile y en algunas de Argentina. Pero debido a la falta de proveedores nacionales, y sumada a las restricciones para su importación, el acceso a este producto es muy dificultoso en nuestro país.

Químicamente, se constituye de un 10,5 \% de magnesio, un 33,5\% de cloro, $52 \%$ de agua y un $4 \%$ de impurezas. Es de color blanco y grasoso al tacto por su gran contenido de humedad. Para aumentar la eficiencia de este producto se sugiere la colocación previa de una carpeta compuesta por la mezcla de coluvio con cloruro de sodio. 
Se recomienda aplicar con una densidad de $1,25 \mathrm{~g} / \mathrm{cm}^{3}$, obtenida por una proporción de 1,5 a 1 en peso de agua, y que el camión regador circule a una velocidad aproximada de 30 a $35 \mathrm{~km} / \mathrm{h}$, lo que resulta en unos $4 \mathrm{l} / \mathrm{m}^{2}$. Para su mantenimiento basta con un riego al día realizado preferentemente de noche (para aprovechar al máximo la propiedad higroscópica que aporta esta solución). Para lo obtención de la densidad deseada se utiliza una motobomba que comete la recirculación de la solución salina.

Se realizó la dilución de $56000 \mathrm{~kg}$ de bischofita en 50000 I de agua y se recirculó hasta obtener una densidad de $1,2 \mathrm{~g} / \mathrm{cm}^{3}$. Se utilizó para el riego 25000 I de esta solución para aplicarlo en una superficie de aproximadamente $1 \mathrm{~km}$ de longitud y $16 \mathrm{~m}$ de ancho. Previo al riego, para preparar la superficie de aplicación, se realizaron trabajos de preparación de la superficie del camino con suelo-sal.

El riego se realizó a una velocidad de $30-35 \mathrm{~km} / \mathrm{h}$ a todo el ancho y tanto en las zonas que tenían un tratamiento con suelo-sal como en las que no lo tenían para poder diferenciar la eficiencia del producto según el tratamiento de la superficie. Se estimó el consumo utilizado por metro cuadrado y fue de aproximadamente $1,56 \mathrm{l} / \mathrm{m}^{2}$.

\subsection{Software Comet}

Para medir y controlar si el uso de aditivos para la disminución de polvo en términos ambientales era o no realmente efectiva, se empleó el Software Comet, el cual está integrado a un equipo captador de polvo (E-Sampler) que combina dos sistemas para determinar las partículas en el aire. Uno de los sistemas efectúa la detección de partículas por medio de un láser (realizando cuarenta mediciones por segundo y promediándolas) y otro de los sistemas utiliza el método gravimétrico. La Figura 2 muestra el esquema del equipo de determinación de polvo por láser. 


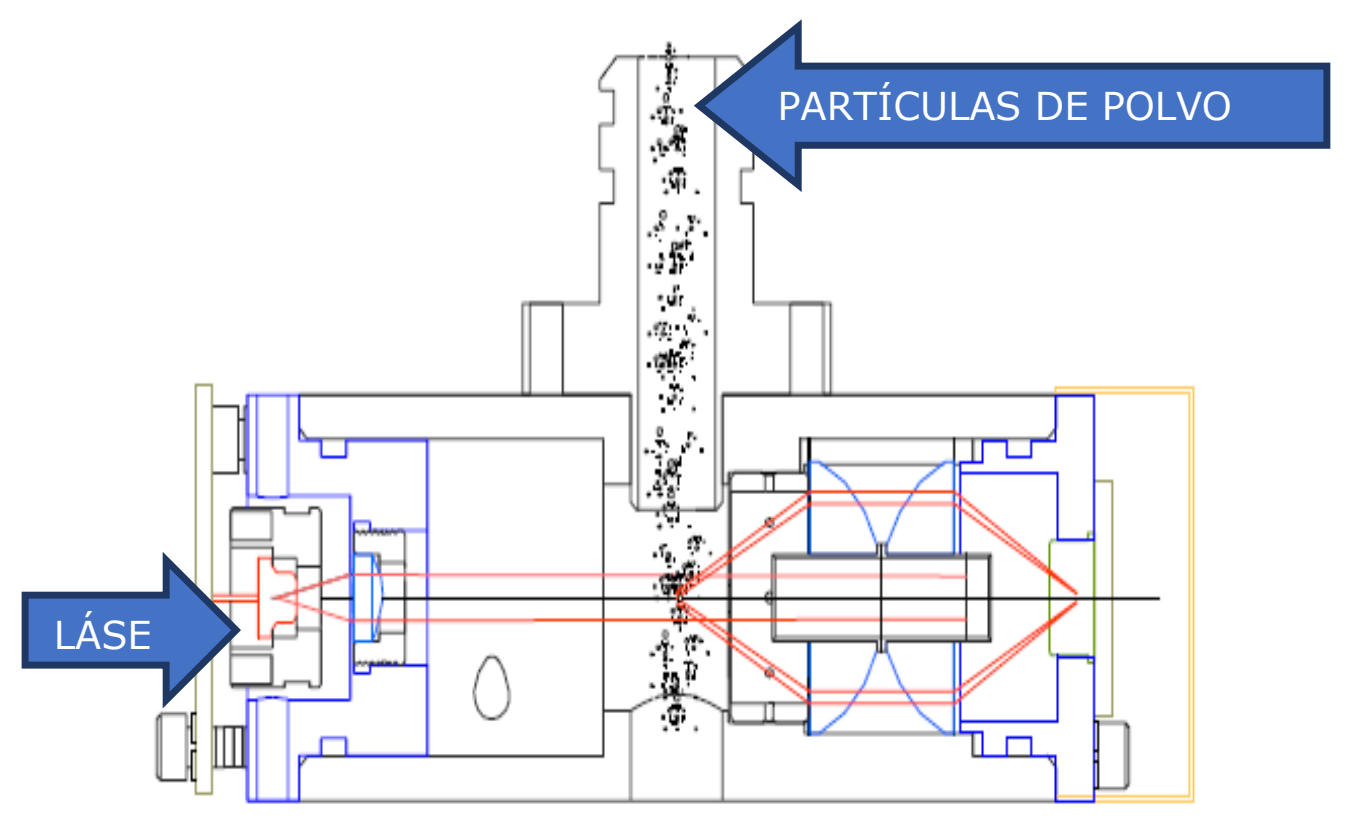

Figura 2. Laser para captación de polvo.

Los datos proporcionados por el equipo son:

- Tiempo, en el que puede verse la fecha y hora.

- Concentración, proporcionada en miligramos por metros cúbicos (pueden cambiarse las unidades de medida).

- Flujo, el flujo óptimo de captación debe ser de $2 \mathrm{l} / \mathrm{m}$.

- Temperatura, en grados centígrados.

- Presión, en pascales.

- $\mathrm{RHx}(\%)$, es el porcentaje de humedad del polvo dentro del captador (el instrumento cuenta con una cámara donde modifica la humedad del polvo que ingresa a un valor constante para evitar errores por diferencia de humedad).

- $\mathrm{RHi}(\%)$, es el porcentaje de humedad con el que el polvo ingresa al captador.

- Ws (m/s), velocidad del viento.

- WD (deg), ángulo de dirección del viento.

- $\mathrm{BV}(\mathrm{v})$, tensión del equipo en voltio, varía entre 0 y 14,45 V.

- Alarm, es una alarma del instrumento, de permanecer en 0 el equipo no manifiesta ningún problema. 
Se utiliza el software Comet para la descarga de datos obtenidos por medio del captador de polvo y luego se realiza por medio de Excel el análisis estadístico de los datos obtenidos, calculando máximos y promedios. El siguiente esquema que se observa en la Figura 3 muestra la metodología utilizada:

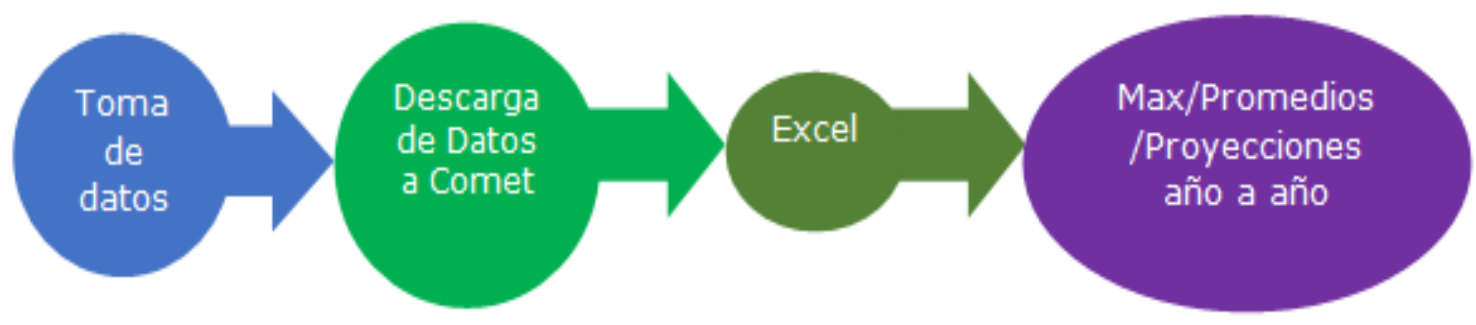

Figura 3. Esquema de la metodología para el análisis de datos.

El programa Excel proporciona una buena portabilidad y facilidad para hacer análisis estadístico diario, mensual y anual; efectuar comparaciones de manera rápida y sencilla entre valores y construir gráficas de interés.

\section{RESULTADOS Y DISCUSIÓN}

\subsection{Melaza}

La aglomeración de partículas generadas a partir del riego perduró hasta 16 días, sin observarse grandes pérdidas luego de la generación de ondas expansivas de voladura y posteriormente de una caída de nieve (debido a que gran porcentaje de la nieve sublima y no se derrite). En la Figura 4 se puede percibir la costra generada al secar la melaza.

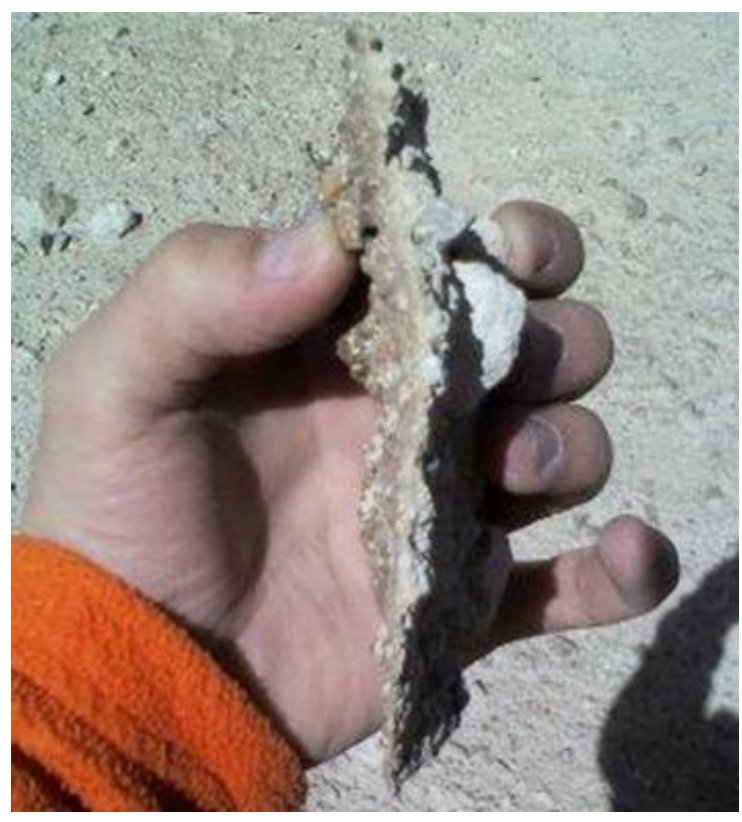

Figura 4. Costra generada a partir de la aplicación de melaza. 
Al notar resultados alentadores, se ejecutaron pruebas en superficies de caminos transitados, por lo que se realizaron riegos con una cantidad de melaza de aproximadamente el $40 \%$ del volumen del tanque del camión y se observó que el tránsito de camiones no permite la generación de una costra como ocurre en bermas regadas, pero sí proporcionó una aglomeración del terreno que retarda la emisión de polvo entre dos y tres días. En cuanto a la aplicación del coagulante en zonas de circulación de vehículos livianos se observó una duración aproximada de 10 días, utilizando un porcentaje de melaza en volumen del $40 \%$.

Este producto proporcionó una solución efectiva para la aglomeración de partículas, tanto en taludes como en superficie de caminos, conteniendo el polvo por debajo de su aplicación. Si bien para superficie de caminos el consumo de producto es mayor y el tiempo de duración de la aplicación es menor, la aglomeración que provocó sobre Steam Heated (material con fórmula química y comportamiento similar al talco pulverulento) no se pudo lograr con ningún otro producto.

Los resultados, luego de la aplicación hecha en zona ripiada, dieron evidencia de la eficiencia que puede lograrse con este producto en zonas de circuitos de camiones. La versatilidad del producto y su solubilidad en agua permitió el desarrollo de una gran variedad de formas de aplicación.

\subsection{Hidróxido de magnesio, $\mathrm{Mg}(\mathrm{OH})_{2}$}

Con la solución de $4000 \mathrm{~cm}^{3}$ de hidróxido de magnesio en 15 I de agua se realizaron pruebas en sectores de suelo para ver la evolución. Al día siguiente de la aplicación se observaron las zonas de prueba y se evidenció que no se generó costra ni se consolidó la zona de prueba (Figura 5).

Al no obtenerse resultados favorables en la etapa de pruebas con la solución preparada con agua a alta temperatura, dado que el asentamiento del hidróxido fue mayor, se decidió no efectuar la prueba sobre la superficie de los caminos. 


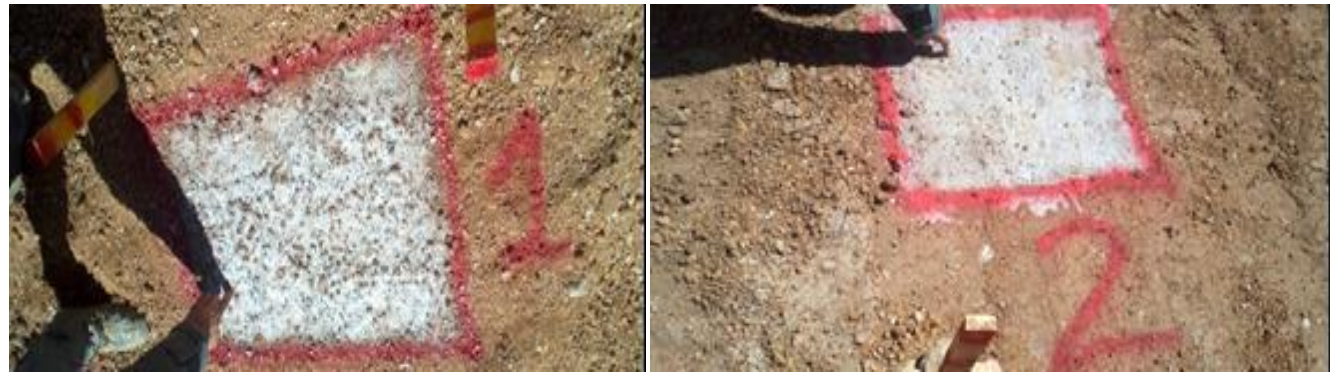

Figura 5. Pruebas en sectores de suelo donde se observa la evolución de la aplicación de hidróxido de magnesio.

Por las pruebas elaboradas se concluyó que al elevar la concentración de hidróxido de magnesio, luego de la agitación, este se mantiene durante más tiempo en suspensión pero la solución nunca llega a ser homogénea. Por ende, al aplicarlo sobre la superficie actúa como hidróxido de magnesio humectado y no como una solución homogénea. Además, la aplicación realizada sobre la superficie demostró que el producto no genera costra ni consolidación de partículas. Finalmente se puede asegurar que el hidróxido de magnesio no es una alternativa para la disminución de polvo en los caminos de la mina.

\subsection{TGC}

El resultado de la aplicación en las tres zonas de prueba de la escombrera se muestra en la Figura 6, donde se puede observar la formación de una costra de aproximadamente $1 \mathrm{~mm}$ de espesor, la cual evita la suspensión de polvo. Debido a la pequeña costra generada y que dicha zona no era blanco de fuertes vientos ni se encontraba cercana a ondas expansivas generadas por voladuras, se decidió descartar el producto por completo para superficie de caminos.

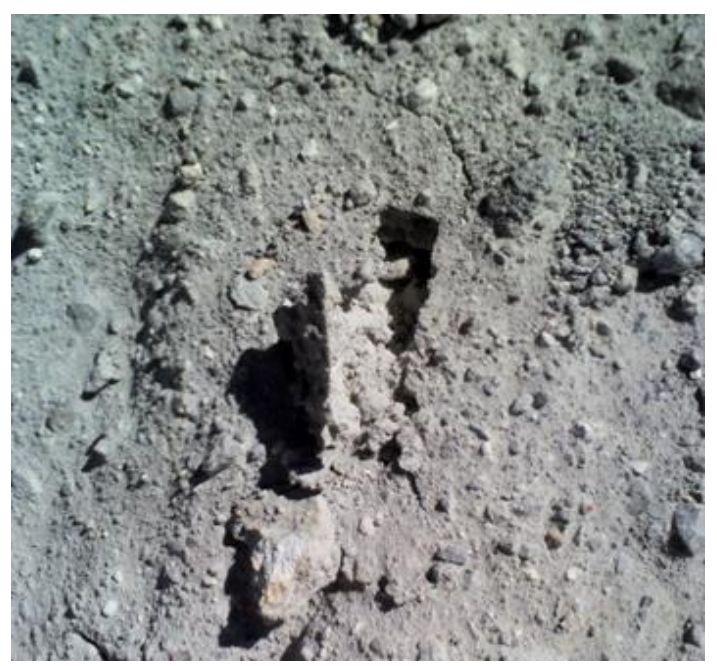

Figura 6. Rotura de costra generada a partir de una leve presión. 
Como la aplicación de este producto generó una costra muy poco resistente a impactos de rocas, se descartó su uso como alternativa viable para la disminución de polvo bajo estas condiciones.

\subsection{Cloruro de sodio, $\mathrm{NaCl}$}

La carpeta creada con suelo-sal se mantiene áspera (Figura 7a), asegurando un buen agarre de los neumáticos, e incluso la humedad podría mejorar las condiciones de tracción de los equipos de mina. La cantidad de sal usada (20 \% volumen) asegura una temperatura más baja de congelamiento de caminos.

Los resultados obtenidos por medio de las aplicaciones de carpetas de suelo sal fueron muy eficaces, aportando una buena consolidación al terreno y evitando la acumulación de nieve sobre la superficie del camino, todo esto a un bajo costo de adquisición y operativo. Sin embargo, este producto solo puede aplicarse en superficies de caminos (tanto transitadas por vehículos livianos como por camiones). Para mejorar su efectividad se recomienda regar con melaza o bischofita sobre su aplicación.

\subsection{Bischofita, $\mathrm{MgCl}_{2} \cdot 6 \mathrm{H}_{2} \mathrm{O}$}

Al día siguiente de la aplicación se pudo observar que el riego con bischofita había dado buenos resultados en toda la zona de aplicación, siendo mejor en aquellas zonas en las que la superficie del suelo tenía un tratamiento previo al riego, con suelo-sal. Dos días después de la aplicación, se observó que la zona trabajada continuaba en buenas condiciones (Figura 7a).

Al nevar se notó que donde se había realizado el trabajo con suelo-sal, previo al regado de bischofita, no había acumulación de nieve. En la Figura $7 \mathrm{~b}$ se muestran los resultados obtenidos. Diez días después, se examinaron nuevamente las zonas y se constató que se mantenían los buenos resultados.

Se pudo verificar que el producto ofreció buenos resultados durante 20 días y luego comenzó a perder eficiencia debido a que se realizó un riego en la zona con una cantidad de agua excesiva, por lo que la combinación del exceso de agua y el peso de los camiones destruyó la capa generada. 


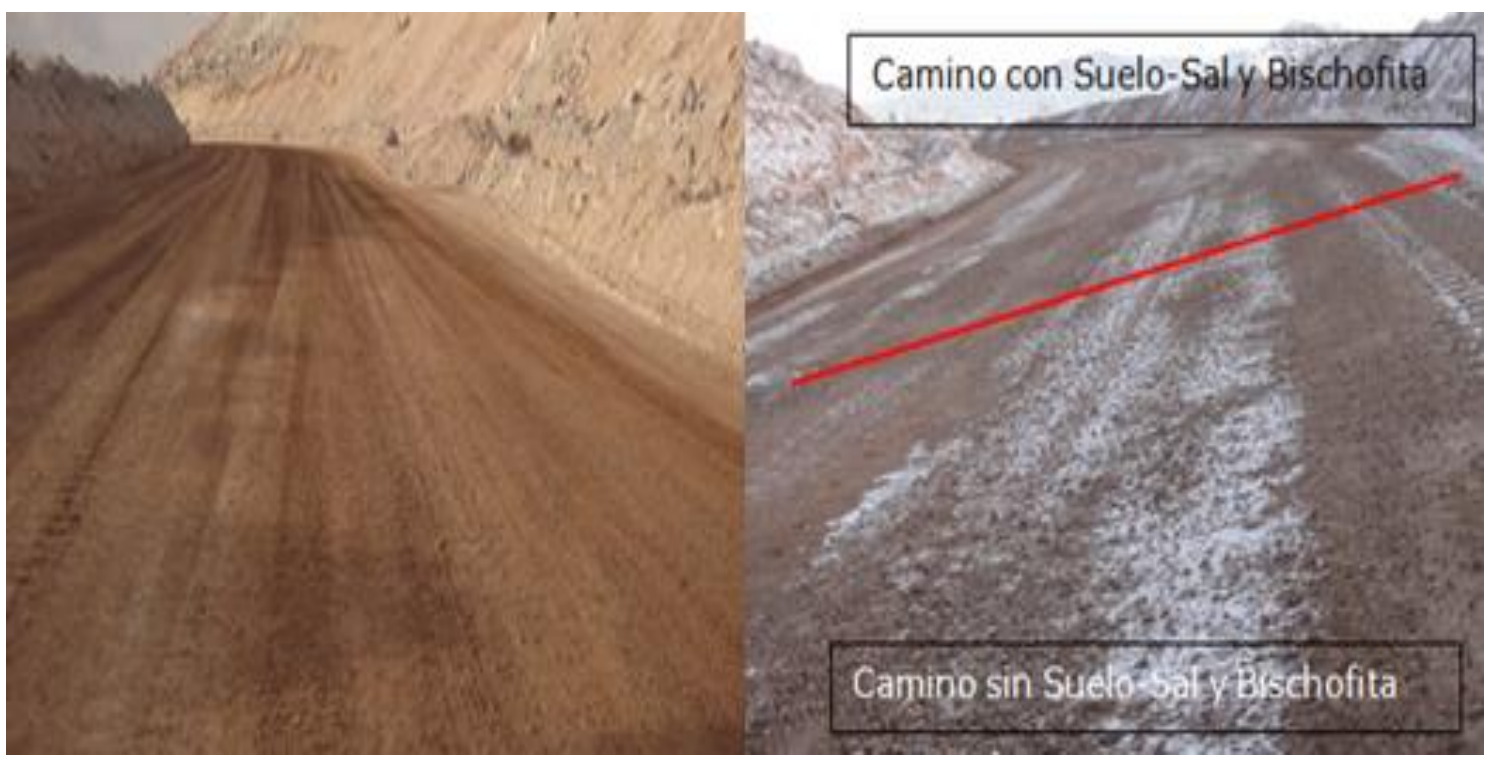

a)

b)

Figura 7. a) Camino con suelo-sal y bischofita. b) Imagen tomada luego de la caída de nieve.

La aplicación de este producto proporciona muy buenos resultados, aumentando su efectividad sobre aquellas zonas tratadas previamente con suelo-sal, lo que permite ahorrar grandes cantidades de agua de riego.

Sin embargo, en caminos destinados al tránsito de camiones se presentan complicaciones por la necesidad de cometer trabajos alternados, debiendo cortar las zonas durante el secado de la aplicación y, por ende, influyendo en los tiempos del transporte de estéril y mineral.

Otro de los grandes inconvenientes es la imposibilidad de aplicarlo durante las épocas níveas debido a la inseguridad que genera por volverse muy resbaloso el camino.

La Tabla 1 ofrece un breve resumen de las aplicaciones realizadas con melaza, TGC, hidróxido de magnesio y bischofita. 
Tabla 1. Cuadro resumen

\begin{tabular}{|c|c|c|c|c|}
\hline Producto & $\begin{array}{l}\text { Fecha de } \\
\text { prueba }\end{array}$ & Zona de prueba & Dilución & Observaciones \\
\hline \multirow{6}{*}{ 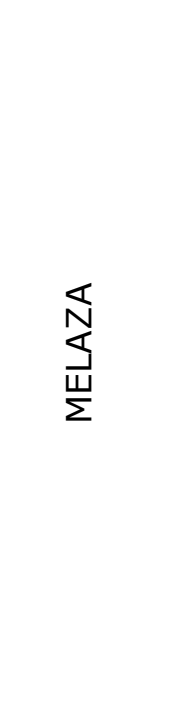 } & $18 / 10 / 2013$ & Taludes & $\begin{array}{l}14 \% \text { en } \\
\text { volumen }\end{array}$ & $\begin{array}{l}16 \text { días de } \\
\text { duración }\end{array}$ \\
\hline & $15 / 04 / 2014$ & Camino de camiones & $\begin{array}{l}40 \% \text { en } \\
\text { volumen }\end{array}$ & 2 - 3 días \\
\hline & $20 / 05 / 2014$ & $\begin{array}{c}\text { Camino de vehículos } \\
\text { livianos }\end{array}$ & $\begin{array}{l}40 \% \text { en } \\
\text { volumen }\end{array}$ & 10 días \\
\hline & $22 / 10 / 2014$ & $\begin{array}{l}\text { Superficie del banco } 4610, \\
\text { sin circulación }\end{array}$ & $\begin{array}{l}35 \% \text { en } \\
\text { volumen }\end{array}$ & más de 16 días \\
\hline & $28 / 12 / 2014$ & $\begin{array}{c}\text { Talud de un botadero } \\
\text { cerrado }\end{array}$ & $\begin{array}{l}30 \% \text { en } \\
\text { volumen }\end{array}$ & más de 60 días \\
\hline & $21 / 02 / 2015$ & Superficie ripiada & $\begin{array}{l}35 \% \text { en } \\
\text { volumen }\end{array}$ & más de 16 días \\
\hline \multirow{4}{*}{ 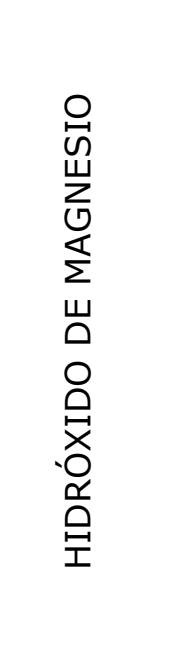 } & $23 / 11 / 2013$ & $\begin{array}{l}\text { Prueba de disolución de } \\
400 \mathrm{~cm}^{3} \text { en } 2 \text { I de agua }\end{array}$ & $\begin{array}{l}20 \% \text { en } \\
\text { volumen }\end{array}$ & $\begin{array}{l}\text { No se pudo } \\
\text { disolver }\end{array}$ \\
\hline & $25 / 11 / 2013$ & $\begin{array}{l}\text { Prueba de disolución de } \\
200 \mathrm{~cm}^{3} \text { en } 2 \text { I de agua }\end{array}$ & $\begin{array}{l}10 \% \text { en } \\
\text { volumen }\end{array}$ & $\begin{array}{l}\text { No se pudo } \\
\text { disolver }\end{array}$ \\
\hline & $25 / 11 / 2013$ & $\begin{array}{c}\text { Prueba de disolución de } 4 \\
000 \mathrm{~cm}^{3} \text { en } 15 \text { I de agua y } \\
\text { prueba de aplicación sobre } \\
\text { superficie }\end{array}$ & $\begin{array}{l}27 \% \text { en } \\
\text { volumen }\end{array}$ & $\begin{array}{l}\text { No se pudo } \\
\text { disolver y no } \\
\text { generó costra ni } \\
\text { consolidó la } \\
\text { superficie }\end{array}$ \\
\hline & $26 / 11 / 2013$ & $\begin{array}{l}\text { Prueba de disolución de } \\
200 \mathrm{~cm}^{3} \text { y } 400 \mathrm{~cm}^{3} \text { en } 2 \text { I } \\
\text { de agua caliente }\end{array}$ & $\begin{array}{l}10 \% \text { y } 20 \% \text { en } \\
\text { volumen }\end{array}$ & $\begin{array}{l}\text { No se pudo } \\
\text { disolver }\end{array}$ \\
\hline$\underset{\cup}{\bigcup}$ & $06 / 11 / 2013$ & Botadero Pampa del Rulo & $3 \%$ & 7 días \\
\hline \multirow{3}{*}{ 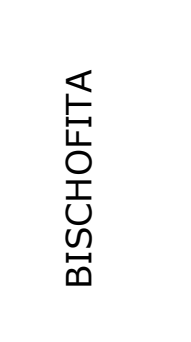 } & $26 / 09 / 2013$ & $\begin{array}{c}\text { CL } 1-\text { CL } 2 \\
\text { (circulación de camiones) }\end{array}$ & $1,2 \mathrm{~g} / \mathrm{cm}^{3}$ & 20 días \\
\hline & $05 / 11 / 2013$ & Camino de camiones & $1,2 \mathrm{~g} / \mathrm{cm}^{3}$ & 12 días \\
\hline & $16 / 11 / 2013$ & $\begin{array}{c}\text { Camino de vehículos } \\
\text { livianos }\end{array}$ & $1,2 \mathrm{~g} / \mathrm{cm}^{3}$ & 21 días \\
\hline
\end{tabular}




\section{CONCLUSIONES}

- Al evaluar el efecto de contención de polvo y su duración en diferentes condiciones climáticas con los productos melaza, hidróxido de magnesio, TGC, cloruro de sodio y bischofita se concluye que el producto bischofita ofrece los mejores resultados en las zonas tratadas previamente con suelo-sal; sin embargo, no es factible aplicarlo durante las épocas de nevada por la inseguridad que genera al tornar muy resbalosos los caminos

\section{REFERENCIAS}

Addo, J.; Sanders, T. y Chenard, M. 2004: Road Dust Suppression: Effect on Maintenance Stability, Safety and the Environment, Phases 1-3. MPC Report No. 04-156. 74 p.

Beaulieu, L.; Pierre, P.; Juneau, S. y Lérege, G. 2011: Maintenance Guide for Unpaved Roads, A Selection Method for Dust Suppressants and Stabilizers. Natural Resources Canada, p. 51.

Gastañaga-Coll, A. y Medina, A. Y. 1963: Control del contaminante polvo en minas y plantas concentradoras. Lima, Perú: Editorial Instituto de Salud Ocupacional.

Guivar-Pereda, P. M. y Zelada-Alaya, R. 2018: Estudio comparativo de supresores de polvo Dasaut, DL10 Plus y Knockout Dustply para la mitigación de material particulado en vías Yanacoha 2018. Tesis de pregrado. Universidad Privada del Norte. Perú. Consultado: 18/12/2018. Disponible en: http://repositorio.upn.edu.pe/bitstream/handle/11537/13955/Gui var\%20Pereda\%20Paul\%20Miki\%20$\%$ 20Zelada\%20Alaya\%20Ronald.pdf?sequence $=1$ \&isAllowed=y

Gutiérrez-Montes, C. A. 2010: Estabilización química de carreteras no pavimentadas en el Perú y ventajas comparativas del cloruro de magnesio (bischofita) frente al cloruro de calcio. Tesis de pregrado. Universidad Ricardo Palma. Perú. Consultado: 18/12/2018. Disponible en: http://repositorio.urp.edu.pe/bitstream/handle/urp/116/gutierrez _ca. pdf? sequence $=1$ \&isAllowed $=y$

Hernández, C. V. 2018: Control de polvo en minería: Tecnología, seguridad y eficiencia. Construcción Minera ( $n$ 04). Consultado: 12/10/2017. Disponible en: http://www.construccionminera.cl/control-de-polvo-en-mineriatecnologia-seguridad-y-eficiencia/\#.W6jV43tKjcc 
Instituto Geológico y Minero de España. 2004: Manual de restauración de terrenos y evaluación de impactos ambientales en minería. 5 ed. Madrid: Instituto Geológico y Minero de España. 360 p. ISBN: 8478400192.

López-Chegne, V. A. 2012: Soluciones anti polvo y lodo. Seguridad minera, 58. Consultado: 25/10/2017. Disponible en: http://revistaseguridadminera.com/salud-ocupacional/solucionesanti-polvo-y-lodo/.

Ospina, J. M.; González, N. M. y Fernández, L. J. 2011: Evidencia temprana de alteración funcional por exposición respiratoria: minería artesanal del carbón en Paipa, Colombia. Revista Facultad Nacional de Salud Pública, 29(4): 445-453. Consultado: 10/09/2018. Disponible

en: http://www.redalyc.org/pdf/120/12021522010.pdf

Ovalle-Cárdenas, E. F. 2014: Estabilización química de los bordes de un terraplén erosionados por escorrentías. Tesis de pregrado. Universidad Austral de Chile, Valdivia, Chile. Consultado: 18/09/2018. Disponible en: http://cybertesis.uach.cl/tesis/uach/2014/bmfcio.96p/doc/bmfcio. 96p.pdf

Pradena, M.; Mery, J. P. y Novoa, E. 2010: Estabilización y mantenimiento de caminos no pavimentados sometidos a condiciones de hielo-nieve en zona de montaña. Revista de la Construcción, 9(2): 97-107. Consultado: 10/09/2018. Disponible en: http://www.redalyc.org/articulo.oa?id=127619215010

Rada-Jaman, R. 2017: Control de polvo: lo barato cuesta caro. Nueva Minería y Energía, 25 de abril. Consultado: 28/08/2018. Disponible en: http://www.nuevamineria.com/revista/control-depolvo-lo-barato-cuesta-caro/

Sistema de Información Geológica Ambiental Minera. Cartas Minerometalogenética $250 \mathrm{~K}$ [mapa en línea]. Consultado: 18/09/2018. Disponible en: http://sigam.segemar.gov.ar/visor/.

Tapia, D. 2017: Control de polvo en minería: A la vanguardia en procesos y tecnología. Nueva Minería y Energía, 7 de mayo. Consultado: 28/08/2018. Disponible en: http://www.nuevamineria.com/revista/control-de-polvo-enmineria-a-la-vanguardia-en-procesos-y-tecnologia/

Ulate-Castillo, A. y Vargas-Sobrado, C. 2018: Control de polvo en caminos no pavimentados. Boletín Técnico Pitra-LanammeUCR, 
9(6), Costa Rica. Consultado: 18/12/2018. Disponible en: https://www.lanamme.ucr.ac.cr/repositorio/bitstream/handle/506 25112500/1049/Bolet\%C3\%ADn\%2006-

$2018 \% 20$ Control\%20de\%20polvo $\% 20$ en $\% 20$ caminos $\% 20$ no $\% 20$ pavimentados. pdf? sequence $=1$ \&isAllowed $=y$

Urteaga, M. P. 2016: Elaboración de un estudio técnico y económico basado en la aplicación del supresor R42-10 para optimizar la frecuencia de regado en la vía de servicios de minera Yanacocha, Cajamarca, Perú. Universidad Privada del Norte, Cajamarca.

Vergara-Ravanal, R. A. 2011: Estabilización y control de polvo con bischofita (cloruro de magnesio hexahidratado). Tesis de pregrado. Universidad Andrés Bello, Santiago, Chile.

Yang, S. C. y Lin, Y. F. 2009: Airway function and respiratory resistance in taiwanese coal workers with simple pneumoconiosis. Chang Gung Med J., 32(4): 438-46.

Recibido: 24/09/2018

Aceptado: 09/11/2018 